\title{
LOCUS DE CONTROL, AUTOEFICACIA PARA LA MEMORIA EPISÓDICA VERBAL Y RENDIMIENTO EN ADULTOS MAYORES NO DEPENDIENTES
}

\section{CONTROL BELIEFS, AGING AND VERBAL MEMORY SELF- EFFICACY IN INDEPENDENT OLDER ADULTS}

\author{
Carolina Feldberg ${ }^{1}$, Dorina Stefan ${ }^{2}$ \\ Instituto de Neurociencias Buenos Aires (INEBA) \\ Consejo Nacional de Investigaciones Cientificas y Técnicas (CONiCET), Buenos Aires, Argentina \\ (RECIBIDO: 4/02/2009 ACEPTADO: 17/06/2009)
}

\begin{abstract}
RESUMEN
Objetivo: En el presente estudio se evalúa la probable relación existente entre el rendimiento del adulto mayor de 65 o más años de edad en tareas de memoria episódica verbal, sus creencias de expectativas de resultado y la autoeficacia en esta función cognitiva. Hipótesis: Las creencias de control y de autoeficacia en tareas de memoria episódica verbal de los adultos mayores no dependientes influyen en su desempeño en actividades que implican esta función cognitiva. Metodología: Se siguieron los pasos de un estudio ex post facto, de fenómenos complejos multicausales, de corte transversal. Fueron seleccionadas las pruebas neuropsicológicas "Rey Auditory-Verbal Learning Test (RAVLT)" y "Subprueba de la Batería de Memoria de Signoret: Recuerdo de una Historia" que evalúan este sistema de memoria, el "Inventario de Autoeficacia para la Memoria Episódica Verbal (IA-MEV)" y dos preguntas que evalúan el locus de control. Estos instrumentos fueron administrados a 200 senescentes que residen en la Ciudad Autónoma de Buenos Aires, Argentina. Conclusiones: El locus de control interno y las dimensiones Nivel y Fuerza de las creencias de autoeficacia en memoria episódica verbal influyen sobre el rendimiento en tareas que implican el uso de esta función cognitiva. Las personas de edad con control en su desempeño en memoria episódica verbal, con una estimación positiva de sus capacidades para resolver estas tareas y una alta confianza en poder hacerlo, se desempeñarán en forma más exitosa en esta función cognitiva que aquellos con un sentimiento de pérdida de dominio, negativa percepción de sus capacidades y baja confianza.
\end{abstract}

Palabras clave: Locus de control, autoeficacia, rendimiento, memoria, vejez.

1 Licenciada en Psicología (UBA), Neuropsicóloga (UBA), PhD UBA, Becaria Postdoctoral del CONICET en INEBA (Instituto de Neurociencias Buenos Aires). E-mail: cfeldberg@ineba.net

2 Licenciada en Sociología (UBA), PhD UBA, Investigador Principal del CONICET en el Instituto de Neurociencias Buenos Aires), Profesora adjunta, Facultad Psicología, UBA. E-mail: dstefani@ineba.net 


\begin{abstract}
Objective: In the present study it was be evaluated the incidence that control beliefs and memory self efficacy have on verbal memory tasks. Hypothesis: Control and memory self efficacy beliefs of older adults affect performance on memory tasks. Method: It was used a transversal ex post facto-comparison group design. The following instruments were selected: Rey Auditory Verbal Learnig Test and Logic Memory , from the Signoret Battery. The SelfEfficacy episodic memory and two questions where added to asses locus of control. There were assessed 200 senescent that reside in the Independent City of Buenos Aires. Discussion: From the values obtained in the statistical analysis made, it is possible to concluded with a certain margin of error, that control and verbal memory self efficacy on episodic memory performance beliefs in older adults, especially internal locus of control with the dimensions self efficacy level and strength, affect performance on memory tasks.
\end{abstract}

Keywords: Control beliefs, performance, aging, memory-self efficacy.

\title{
INTRODUCCIÓN
}

Los diferentes estudios realizados acerca de la declinación intelectual que se produce como consecuencia del proceso de envejecimiento, se han centrado mayoritariamente en la identificación de indicadores objetivos. Sin embargo, las apreciaciones subjetivas, como son las expectativas de resultados y las atribuciones de las causas que llevaron a los mismo, juegan un rol central en el proceso de envejecimiento y las funciones cognitivas, ocupando un rol central en las teorías de la autoeficacia y de la desesperanza aprendida (Lachman \& Jelalian, 1984; West \& Yassuda, 2004).

\section{Locus de control y memoria en adultos mayores}

La teoría del control sostiene que los sujetos pueden tener diferentes reacciones en iguales circunstancias, de acuerdo a su percepción de dominio en los siguientes tres aspectos: la motivación, la cognición y la emoción. La sensación de control o falta de ella surge de la interacción de los elementos de esta tríada. Asimismo, estos supuestos derivan de la "Teoría de la Desesperanza Aprendida" y señalan que cuando una persona se siente indefensa, es de vital importancia la atribución de causalidad que ella realiza, lo cual determinará la generalidad o cronicidad de la sensación de desesperanza y su baja autoestima. Por lo que existe una relación entre la sensación de desesperanza y el locus de control (Abramson y Seligman, 1978).

Las atribuciones de causalidad que las personas realizan frente a un acontecimiento pueden dividirse en dos dimensiones: internas o externas. Son internas cuando las personas tienden a pensar que los resultados de un acontecimiento se deben a las respuestas que ellos efectuaron; mientras que la atribución es externa cuando los sujetos creen que las causas se refieren a factores ajenos a ellos, como, por ejemplo, la suerte o el destino. Asimismo, la apreciación de causalidad refleja de forma indirecta las teorías implícitas que la persona tiene respecto de la naturaleza de la memoria y cómo cambia con la edad, así como también la posibilidad de control que se puede ejercer sobre esta función cognitiva (Abramson \& Seligman, 1978). 
Diferentes estudios sugieren que los adultos mayores, a diferencia de los adultos jóvenes, tienen una mayor tendencia a atribuir su rendimiento cognitivo a factores internos que externos. Este estilo atribucional con un rendimiento pobre se asocia con la desesperanza aprendida y con la aparición de un estado de ánimo depresivo (Heckhausen \& Schulz, 1995). Otras investigaciones coinciden en señalar las diferencias en las atribuciones de causalidad según la edad y su influencia en la utilización de estrategias en el recuerdo de información en la memoria episódica. Los adultos mayores tienden a utilizar en menor medida estrategias de codificación de la información (Devolder \& Pressley, 1992).

Por su parte, Hertzog, Lineweaver y Mc Guire (1999) plantean que las mejores estrategias para el recuerdo de información incluyen el procesamiento semántico, el uso de estrategias de tipo verbal e imaginería visual, así como también es eficiente el uso de estrategias que tengan en cuenta las características estructurales de las palabras. Estos autores han observado que, aunque se beneficiarían de su uso, un alto número de adultos mayores no utiliza estrategias de codificación óptimas y que su rendimiento cognitivo podría mejorar a través de la incorporación de estas estrategias de organización de la información. Esto compensaría las dificultades producidas en la memoria como resultado de la declinación cognitiva propia de la edad. En consecuencia resulta posible establecer una tríada que sustenta el rendimiento cognitivo en los adultos mayores, la cual está formada por las teorías implícitas acerca del envejecimiento, la atribución de control y el uso de estrategias de organización de la información.

\section{Autoeficacia y memoria en adultos mayores}

Los adultos mayores tienden en forma frecuente a subestimar sus habilidades intelectuales. Al respecto, la teoría de la autoeficacia sugiere que tal comportamiento puede tener graves consecuencias sobre el rendimiento, dado que sostiene que dichas creencias son las que más influyen en la sensación de conducta competente que los seres humanos experimentan y que juegan un papel muy importante en la determinación de las elecciones que ellos realizan, en el esfuerzo que dedicarán y en el tiempo que perseverarán cuando se enfrenten a dificultades. Además, estas creencias resultan ser un buen predictor del grado de ansiedad o confianza que los sujetos experimentan (Rey, Blasco \& Borràs, 2000).

Se define a la autoeficacia como las creencias acerca de la propia capacidad para organizar y ejecutar los cursos de acción necesarios a fin de alcanzar determinados resultados. Distingue tres dimensiones en las creencias de autoeficacia:

a) el nivel: es la estimación personal que efectúa un sujeto sobre cuál es el mejor rendimiento posible para él en un momento y circunstancia determinados.

b) la fuerza: es la confianza que experimenta la persona en cuanto a poder alcanzar dicho rendimiento, $y$

c) la generalidad: es la variación de la autoeficacia desde considerar ser eficaz tan sólo ante algunas circunstancias concretas hasta percibir contar con los recursos necesarios para solucionar cualquier situación (Bandura, 1986). 
Berry, West y Dennehey (1989) señalan que el sistema de la memoria se vuelve menos confiable con la edad. Se produce entonces una sensación de incertidumbre en torno a esta función mnésica y, por lo tanto, a su eficacia; lo que puede inhibir un rendimiento óptimo y dificultar el funcionamiento en tareas de memoria. Este autor enfatiza que el conocimiento que uno tiene sobre las propias capacidades es también una faceta importante de la metacognición, y que los procesos cognitivos, afectivos y motivacionales, se activan según su naturaleza, a través de las creencias sobre la propia eficacia y pueden mejorar o empeorar el rendimiento en tareas que implican el uso de esta función cognitiva.

En consecuencia, a partir del enfoque de Bandura (1997), los adultos mayores, que creen tener algún control sobre el funcionamiento de su memoria, deberían beneficiarse por estas creencias y mostrarse más motivados para invertir tiempo y esfuerzo en el aprendizaje de nueva información, al mismo tiempo que utilizarán estrategias de codificación de forma más conciente. Por el contrario, aquellas personas que no utilizan estrategias de organización de la información, generalmente, creen que la memoria es un trazo estable y que cualquier intento por guiarla o controlarla es en vano. Sumado a esto, las personas que tienen una alta autoeficacia suelen utilizar un mayor esfuerzo y ser más persistentes cuando intentan recordar nueva información.

\section{Objetivo}

El propósito de este estudio es evaluar en adultos mayores la probable relación existente entre las creencias de control, las de autoeficacia para la memoria episódica verbal y el rendimiento en tareas que implican esta función cognitiva.

\section{Hipótesis}

Las creencias de dominio y las de autoeficacia en memoria episódica verbal de las personas de edad inciden en su rendimiento en tareas en las que se utilizó este sistema mnésico.

\section{MÉTODO}

\section{Diseño}

En este estudio no experimental, se siguieron los pasos de un estudio ex post facto, de fenómenos complejos multicausales, de corte transversal.

\section{Población y muestra}

La población objetivo del estudio se refiere a adultos mayores de 65 o más años de edad, autoválidos, de diferente género, de nivel socioeconómico medio, que no presentan trastornos cognitivos ni estado depresivo y que habitan en sus hogares en un gran centro urbano como es la Ciudad Autónoma de Buenos Aires, Argentina.

En lo concerniente a la muestra, en un primer momento las unidades muestrales fueron seleccionadas siguiendo la estrategia de muestreo no probabilístico de tipo accidental. Dado 
que existe en este grupo etario una alta incidencia de alteraciones patológicas que afectan a la memoria, se consideró importante para la selección de los sujetos que finalmente integrarían la muestra que cumplieran con los siguientes criterios de inclusión: 1) debían obtener en el Mini Mental State Examination (MMSE), un puntaje superior que, de acuerdo a los años de escolaridad, oscilaba entre 24 y 27 puntos, 2) en la Escala de Depresión Geriátrica (GDS), los puntajes debían ser menores a los 10 puntos, y 3) las respuestas al Cuestionario de Antecedentes Neurológicos debían ser negativas. De este modo, se efectúa el screening general de las funciones cognitivas, de la existencia o no de depresión y de patologías neurológicas, quedando finalmente la muestra conformada por 200 sujetos, cuya edad promedio era de 72.44 años y el mayor porcentaje de los casos se ubicaba en el género femenino (76.5), estado civil viudo (48\%), nacionalidad argentina (82\%) y estudios primarios completos (36.5\%). Por último, las ocupaciones principales antes de jubilarse fueron las de empleado (36\%), oficios varios $(22 \%)$, comerciante $(16 \%)$ y profesional independiente (15\%), que junto con el nivel educacional de los participantes indican que todos los adultos mayores pertenecían al nivel socioeconómico medio ${ }^{1}$.

\section{Material y procedimientos}

\section{a) Instrumentos de medición}

1. Screening de las funciones cognitivas del sujeto

- Mini Mental State Examination (MMSE) de Folstein (Spreen y Strauss, 1998)

El objetivo de este test es hacer un screening del deterioro mental, especialmente en los adultos mayores. Evalúa el deterioro cognitivo; es útil en la detección de cambios intelectuales que ocurren en el tiempo. Es muy utilizado porque es un test corto, fácil de administrar y de puntuar.

El test está compuesto de varios ítems, que son: evaluación, orientación en tiempo y espacio, atención concentración, lenguaje (comprensión y expresión), habilidad constructiva, recuerdo inmediato y recuerdo diferido.

Puntuación: Se obtiene un total de respuestas correctas que es igual a 30 puntos. Cualquier falla en la respuesta es considerada un error.

La consistencia interna del instrumento fue evaluada a través del procedimiento alfa de Cronbach, arrojando valores que van de .31 para sujetos sanos a .96 para pacientes con diferentes patologías.

\section{- Escala de Depresión Geriátrica (GDS) (Spreen y Strauss, 1998)}

Se utilizó esta escala, para evaluar la presencia o no de depresión. Consta de 30 ítems, los cuales fueron seleccionados por un grupo de 100 expertos. Los ítems son de respuesta dicotómica (SÍ/No), y resultan de utilidad para distinguir, en términos generales, ancianos deprimidos de no deprimidos. Según estudios realizados en personas de edad, los autores sugieren que una puntuación de 0 a 
10 debe ser considerada normal y puntajes iguales o mayores de 11 deben ser tomados como un posible indicador de depresión.

La confiabilidad del instrumento fue medida a través de las técnicas de alfa de Cronbach y mitades partidas. Se estimó la consistencia interna del instrumento mediante el procedimiento " $\alpha$ " de Cronbach. El valor obtenido en la aplicación de este análisis fue de .94. Por su parte, el análisis de mitades partidas arrojó un valor del Coeficiente "r" de Pearson igual a .94.

- Cuestionario de antecedentes neurológicos (construido "ad hoc" por las autoras)

Este cuestionario recaba información acerca del estado clínico general del paciente, posibilitando la detección de posibles alteraciones neurológicas que podrían disminuir su rendimiento mnésico.

2. Luego de haber realizado el screening inicial a los sujetos seleccionados en un primer momento, a los 200 elegidos se les administró un cuestionario de datos básicos y diversas pruebas neuropsicológicas y psicosociales, las cuales fueron seleccionadas de acuerdo a sus características psicométricas satisfactorias para este tipo de estudio y por su fácil administración y evaluación.

- Cuestionario de Datos Básicos: consta de 11 preguntas cerradas con alternativas fijas que recaban información del adulto mayor acerca de las variables sociodemográficas edad, sexo, nacionalidad, estado civil, educación y ocupación. Asimismo, dos preguntas de igual característica informan acerca de las creencias del estado de la memoria y del estado de salud.

\section{Pruebas neuropsicológicas}

Para la evaluación del rendimiento objetivo en tareas de Memoria Episódica Verbal, fueron seleccionadas las siguientes pruebas neuropsicológicas:

\section{- $\quad$ Rey Auditory-Verbal Learning Test (RAVLT) (Spreen y Strauss, 1998)}

Esta es una prueba de lápiz y papel, corta y de fácil administración que evalúa "span" de memoria inmediata, proporciona una Curva de Aprendizaje, revela "estrategias de aprendizaje" (o su ausencia) y detecta tendencias a la interferencia retroactiva y proactiva, a la confusión y a la confabulación en tareas de memoria. Mide, además, retención de Corto y Largo Término, seguidas de una actividad intercalada, y permite hacer una comparación entre eficiencia en la recuperación y aprendizaje.

Se estimó la consistencia interna del instrumento mediante el procedimiento " $\alpha$ " de Cronbach, obteniéndose un valor igual a .90 .

Puntuación: Se obtienen diferentes puntajes; un puntaje total que surge de la suma de los resultados en los ítems de I-V, un puntaje de Recuerdo luego de una interferencia, que se denomina Recuerdo Inmediato, y un puntaje de Recuerdo Diferido y de Reconocimiento de una Lista de Palabras. 
- Subprueba de la Batería de Memoria de Signoret: Recuerdo de una Historia (Lezak, 1995)

Esta prueba consiste en el relato de una historia, la cual contiene 12 ideas centrales que deben ser recordadas por el sujeto evaluado. Consta de dos etapas, una de Recuerdo Inmediato, la cual se solicita inmediatamente después de que la historia es narrada, y una etapa de Recuerdo Diferido, la cual se realiza 30 minutos después de que es presentada la historia.

La confiabilidad del instrumento fue medida a través de las técnicas " $\alpha$ " de Cronbach y el valor obtenido fue igual a .64.

Puntuación: El puntaje máximo que puede obtener un sujeto es de 12 puntos en cada una de las instancias de recuerdo. El recuerdo de algunos ítems de la historia equivale a 1 punto y otros a 0.50 .

\section{Pruebas psicosociales}

\section{- Evaluación del Locus de Control}

Para la evaluación de la experiencia de dominio del sujeto entrevistado, se incluyeron dos preguntas. La primera, de tipo cerrada y de respuestas múltiples, permitió indagar respecto de las expectativas que el sujeto tiene acerca de su rendimiento en las pruebas neuropsicológicas que evalúan la memoria episódica verbal. La segunda, de tipo abierta, el sujeto debía atribuir las causas del rendimiento percibido. Las respuestas fueron categorizadas de acuerdo con la clasificación propuesta por Abramson \& Seligman (1978), en internas o externas. Luego fueron recategorizadas de acuerdo con la clasificación de Lachman y Jelalian (1984).

- Inventario de Autoeficacia para la Memoria Episódica Verbal (IA-MEV) (Feldberg, 2008)

Con el propósito de estudiar la autoeficacia de los adultos mayores en tareas de memoria episódica verbal, se utilizó un instrumento específico que evalúa las creencias acerca de la propia capacidad para realizar tareas que impliquen esta función mnésica, en sus dimensiones: Nivel y fuerzas de las creencias, que fue construido "ad hoc". Esta prueba se elaboró sobre la base del Memory Self-efficacy Questionnaire (MSEQ), de Berry et al. (1989), y la Escala de Autoeficacia para la memoria utilizada por Rebok y Balcerak (1989).

Siguiendo la nomenclatura utilizada por Berry et al. (1989), el Inventario proporciona dos valores, un valor que indica el Nivel de la Autoeficacia (NAE) y otro que representa la Fuerza de la Autoeficacia (FAE), para las tareas planteadas en los dos ítems que componen el instrumentos: Recuerdo de una Lista de Compras y Recuerdo de una Historia. Además, de un valor general para ambas dimensiones.

El instrumento posee una adecuada consistencia interna, la cual se estimó mediante el procedimiento de Cronbach. (Pérez, Beltramino \& Cupani, 2003). El puntaje obtenido en la aplicación de este análisis fue de .85 . 


\section{b. Análisis estadístico}

Con el fin de establecer si los resultados obtenidos en este estudio apoyan la hipótesis formulada en el mismo, se aplicó la prueba " $t$ " para muestras independientes, para evaluar si las diferencias existentes entre aquellos sujetos que atribuyeron a la habilidad o falta de ella como causa de su rendimiento a) en las dimensiones Nivel y Fuerza de las creencias de autoeficacia y b) en las pruebas Recuerdo de una Lista de Palabras y Recuerdo de una Historia resultan estadísticamente significativas o se deben al azar. Asimismo, se utilizó esta prueba de significación estadística para evaluar si existen diferencias significativas entre los sujetos con altos y bajos niveles y fuerza de autoeficacia en el rendimiento en las pruebas neuropsicológicas. En todos los casos se fijó un nivel de probabilidad de error menor o igual que .05.

\section{RESULTADOS}

En la Tabla N. ${ }^{o} 1$ se presentan las frecuencias y porcentajes correspondientes a las dimensiones habilidad, esfuerzo, dificultad de la tarea y suerte, de las cuales las dos primeras corresponden a la categoría locus interno y el resto a locus externo de la variable locus de control. Al inspeccionar esta tabla, se observa que el $83.50 \%$ de los adultos mayores atribuyeron a su habilidad o falta de este atributo como causa de su rendimiento, presentando un predominio del locus de control interno en sus respuestas. ${ }^{2}$

Tabla N. ${ }^{0}$ 1. Locus de Control. Atribución de rendimiento.

\begin{tabular}{clcc}
\hline \multirow{2}{*}{ Locus de control } & \multicolumn{2}{c}{ Muestra } \\
\cline { 2 - 4 } & & $\mathbf{n}$ & $\mathbf{\%}$ \\
\hline Interno & Habilidad & 89 & 44.5 \\
$(83.50 \%)$ & Falta de habilidad & 78 & 39 \\
\hline \multirow{3}{*}{ Externo } & Esfuerzo & 9 & 4.5 \\
& Falta de esfuerzo & 6 & 3 \\
$(17.50 \%)$ & Tarea sencilla & 11 & 5.5 \\
& Tarea difícil & 3 & 1.5 \\
& Suerte & 2 & 1.0 \\
& Falta de suerte & 2 & 1.0 \\
\hline & Total & $\mathbf{2 0 0}$ & $\mathbf{1 0 0}$ \\
\hline
\end{tabular}

En la Tabla N. ${ }^{\circ} 2$ se presentan las medias aritméticas (m) y desviaciones estándares (ds) de los puntajes que corresponden a las creencias de dominio y al rendimiento objetivo en las pruebas que evalúan la memoria episódica verbal. En la última columna se presentan los valores "to" observados que correspondes a cada una de las pruebas neuropsicológicas, que revelan la existencia de una relación significativa entre las creencias de dominio y el rendimiento obtenido por los sujetos evaluados en la instancia de recuerdo diferido de la prueba Recuerdo de una Lista de Palabras, pero no entre éstas y el Recuerdo de una Historia. Los adultos mayores creen que la causa de su rendimiento en la prueba RAVLT 
reside en la habilidad o falta de ese atributo para realizar esta tarea, resultando la diferencia existente estadísticamente significativa. Este mismo comportamiento no ocurre con respecto al Recuerdo Diferido de una Historia.

Tabla N. ${ }^{0}$ 2. Rendimiento objetivo según locus de control interno en MEV.

\begin{tabular}{|c|c|c|c|c|c|c|c|}
\hline \multirow{3}{*}{$\begin{array}{c}\text { Pruebas } \\
\text { Neuropsicológicas }\end{array}$} & \multicolumn{6}{|c|}{ Locus de Control Interno } & \multirow{3}{*}{ to } \\
\hline & \multicolumn{3}{|c|}{ Falta de Habilidad } & \multicolumn{3}{|c|}{ Habilidad } & \\
\hline & $\mathbf{m}$ & ds & $\mathbf{n}$ & $\mathbf{m}$ & ds & $\mathbf{n}$ & \\
\hline Rec. Lista de Palabras & 6.56 & 2.58 & 78 & 7.72 & 2.28 & 89 & $9.86^{*}$ \\
\hline Recuerdo Historia & 8.07 & 6.89 & 78 & 7.54 & 1.71 & 89 & .05 \\
\hline
\end{tabular}

En la Tabla N. ${ }^{\circ} 3$ se presentan las medias aritméticas y desviaciones estándares de los puntajes de rendimiento en las pruebas neuropsicológicas utilizadas según las dimensiones Nivel y Fuerza de las creencias de autoeficacia. Los valores "to" observados, obtenidos para cada prueba, indican la influencia del rendimiento que los adultos mayores imaginan para sí (Nivel de la autoeficacia) y de la confianza (Fuerza de la autoeficacia) que tienen de poder obtenerlo, sobre el rendimiento en las pruebas neuropsicológicas utilizadas para evaluar la memoria episódica verbal, Recuerdo de una Lista de Palabras y Recuerdo de una Historia.

Tabla N. ${ }^{0}$ 3. Rendimiento objetivo según creencias de autoeficacia. ${ }^{3}$

\begin{tabular}{|c|c|c|c|c|c|c|c|c|c|c|c|c|c|c|}
\hline \multirow{4}{*}{$\begin{array}{c}\text { Pruebas } \\
\text { Neuropsic. }\end{array}$} & \multicolumn{14}{|c|}{ Autoeficacia } \\
\hline & \multicolumn{7}{|c|}{ Nivel } & \multicolumn{7}{|c|}{ Fuerza } \\
\hline & & Bajo & & & Alto & & & & Baja & & & Alta & & \\
\hline & m & ds & $\mathbf{n}$ & m & ds & $\mathbf{n}$ & to & m & ds & $\mathbf{n}$ & m & ds & $\mathbf{n}$ & to \\
\hline $\begin{array}{l}\text { Rec.Lista } \\
\text { Palabras }\end{array}$ & 5.50 & 2.41 & 50 & 7.93 & 2.51 & 102 & $32.30 * * *$ & 5.98 & 2.81 & $54-$ & 8.13 & 2.56 & 55 & $17.36^{* * * *}$ \\
\hline $\begin{array}{l}\text { Recuerdo } \\
\text { Historia }\end{array}$ & 6.84 & 1.98 & 90 & 8.14 & 2.00 & 90 & $13.59 * * *$ & 6.57 & 2.20 & 55 & 8.22 & 1.96 & 61 & $18.14 * * *$ \\
\hline
\end{tabular}

En la Tabla N. ${ }^{\circ} 4$ se presentan las medias aritméticas y desviaciones estándares de los puntajes que corresponden a las creencias de autoeficacia según el locus de control interno. En la última columna, los valores "to" observados muestran que para las dimensiones Nivel y Fuerza, los sujetos creen que su estimación de lograr el rendimiento esperado y la confianza en poder hacerlo dependen de la presencia o ausencia de su habilidad. 
Tabla N. ${ }^{\circ}$ 4. Creencias de autoeficacia según locus de control.

\begin{tabular}{lcccccccc}
\hline & \multicolumn{8}{c}{ Locus de Control Interno } \\
\cline { 2 - 9 } Autoeficacia & \multicolumn{3}{c}{ Falta de Habilidad } & \multicolumn{5}{c}{ Habilidad } \\
& $\mathrm{m}$ & $\mathrm{ds}$ & $\mathrm{n}$ & $\mathrm{m}$ & $\mathrm{ds}$ & $\mathrm{n}$ & to \\
\hline Nivel & 62.26 & 15.12 & 78 & 57.50 & 19.94 & 89 & $3.06^{* *}$ \\
Fuerza & 53.33 & 23.20 & 78 & 62.35 & 18.17 & 89 & $7.65^{* *}$ \\
\hline${ }_{* *} p \leq .01$ & & & & & & &
\end{tabular}

\section{DISCUSIÓN}

Una de las principales razones por las cuales los gerontólogos se han interesado en el estudio de la autoeficacia de la memoria y de las atribuciones de control que los ancianos realizan en esta función cognitiva, se debe a que se halló en diversos estudios empíricos que ambos sistemas de creencias afectan los comportamientos relacionados con la memoria (Bandura \& Wood, 1989)

La teoría de la autoeficacia, frente al hecho que los adultos mayores subestiman en forma frecuente sus habilidades cognitivas, plantea que las creencias que sustentan este comportamiento inciden en forma negativa sobre el rendimiento del anciano en tareas que impliquen esta función cognitiva (Perlmutter, 1978). Asimismo, la teoría del control señala que las atribuciones que las personas realizan acerca de las causas de su rendimiento, también son un factor que puede tener severas consecuencias sobre aspectos afectivos y conductuales de la persona de edad. Un mayor sentido de control sobre el rendimiento cognitivo puede llevar a la persona de edad a un mayor esfuerzo en la identificación y aplicación de estrategias de codificación de la información, lo que daría como resultado un mejor rendimiento. Ya sea que la apreciación de control interno sea positiva o negativa, el hecho de adjudicar su rendimiento en tareas de memoria a un factor interno determinaría que la persona de edad asuma una mayor responsabilidad en su ejecución, lo que produciría la aparición de estrategias de afrontamiento que permiten compensar alguna dificultad experimentada (Miller \& Lachman, 2000; Verhaeghen, Geraerts \& Marchen, 2000).

Por otra parte, Mobility, Lemke y Grisisn (1991) consideran a las personas de edad como el segmento de población con mayor riesgo de perder el sentido de control en relación a su ambiente. Frente a las sucesivas pérdidas de roles significativos, las oportunidades de ejercer control disminuyen. De acuerdo con estos autores, para evitar esta situación, las personas ancianas deben identificar y realizar actividades que tiendan a aumentar su percepción de control personal y eficacia. Las actividades vividas por los sujetos como gratificantes son en general las que tienden a producir percepciones de capacidad personal, control y a la vez, eficacia en su desempeño. 


\section{CONCLUSIONES}

Los resultados obtenidos en el presente estudio son consistentes con lo anteriormente expuesto y apoyan la hipótesis formulada, pero restringida al locus de control interno por restricciones estadísticas acerca de la distribución de frecuencias de la variable Locus de Control. La experiencia de dominio del anciano y la dimensión Nivel y Fuerza de las creencias de autoeficacia en memoria episódica verbal, afectan su rendimiento en la prueba Recuerdo de una Lista de Palabras. La gran mayoría de los participantes del estudio $(83,50 \%)$ atribuyó como causa de su rendimiento en actividades con memoria episódica verbal a la presencia o ausencia de habilidad para dicha tarea cognitiva, consideradas de locus de control interno. A lo que se suma que los adultos mayores con una percepción positiva de sus capacidades y $\mathrm{n}$ alta confianza en lograr el éxito, eligen, más probablemente, la opción de intentar el control sobre el funcionamiento de su memoria, cuando se presente como una posibilidad (Hertzog et al., 1998; De Frias, Dixon y Bäckman, 2003).

Los recursos psicosociales son la principal fuente que les facilitará a los adultos mayores compensar y adaptarse a la declinación experimentada en la esfera cognitiva. Cuán bien las personas de edad puedan o no adaptarse a los cambios que ocurren en dominios tales como la memoria, dependerá del conocimiento que tengan acerca de sí mismas y de las creencias autorreferenciales que ellas posean. Esto les permitirá enfrentar saludablemente los cambios producidos por el paso del tiempo.

Se continuará con esta línea de investigación, evaluando los estilos de afrontamiento de las personas de edad. Pearman y Storandt (2004) aducen que un afrontamiento activo ante los problemas y conflictos determinará, con mayor probabilidad, que la persona de edad acepte y se esfuerce para efectuar mejor la tarea, exhibiendo su potencial y tendiendo a participar en actividades sociales en las que se utilice la memoria episódica, resguardando de este modo su bienestar general.

\section{NOTAS}

1. La ubicación de los sujetos en la categoría Nivel Económico Social Medio fue realizada a partir de los puntajes obtenidos en una versión del Índice de Nivel Económico Social de Gino Germani (Grimson, Blanco, Estrugamou, Lastres, Henchí \& Philipott, 1972).

2. En los análisis estadísticos posteriores se tuvo sólo en cuenta la dimensión "habilidad "de locus de control "interno", dado que el resto de las categorías presentan porcentajes menores al $10 \%$.

3. A los fines de este análisis fue eliminada las categorías intermedias respectivas de las dimensiones Nivel y Fuerza de las creencias de autoeficacia.

\section{AGRADECIMIENTO}

Las autoras agradecen a las autoridades de INEBA el interés y apoyo brindados para la realización de este estudio que se llevó a cabo en esta institución. 


\section{REFERENCIAS BIBLIOGRÁFICAS}

1. Abramson L.Y. \& Seligman M.E.P. (1978). Learned helplessness in humans: critique and reformulation. Journal of Abnormal Psychology 87, (1):49-74.

2. Bandura, A. (1997). Self-efficacy: The exercise of control. New York: Freeman.

3. Bandura, A. \& Wood, R. (1989). Effect of perceived controllability and performance standards on self- regulation of complex decision making. Journal of Personality and Social Psychology, 56 (5): 805-814.

4. Bandura, A. (1986). Social foundations of thought and action: a social cognitive theory. NJ: Prentice Hall.

5. Berry, J.M., West, R.L. \& Dennehey, D.M. (1989). Reliability and validity of the memory self efficacy questionnaire. Developmental Psychology, 25(5): 701-713.

6. De Frias, C.M., Dixon, R.\& Bäckman, L. (2003). Use of memory compensation strategies is related to psycholosocial and health indicators. Journals of Gerontology, 58B (1): 12-22.

7. Devolder, P.A. \& Pressley, M. (1992). Causal attributions and strategy use in relation to memory performance differences in younger and older adults. Applied Cognitive Psychology, 6:629-642.

8. Feldberg Carolina (2008). Inventario de autoeficacia para la memoria episódica verbal. Revista Neurológica Argentina; 33: 44-49.

9. Heckhausen J., \& Schulz R.(1995). A life span theory of control. Psychological Review vol 102(2), 284-304.

10. Hertzog, C., Lineweaver T.T., Mc Guire C.L. (1999). Social cognition and aging. New York: Ed. American Press.

11. Grimson, W.R.; Blanco, A.C.; Estragamou, M; Lastres, E.; Necchi, s; Phillpott, E.; Tesaire, A.M. (1972). Investigaciones epidemiológicas de entidades psiquiátricas. Boletín Oficina Sanitaria Panamericana, 79, 572.

12. Lachman M.E.,\& Jelalian E. (1984). Self-efficacy and attributions for intellectual performance in young and elderly. Journal of Gerontology vol 39 (5), 577-582.

13. Lezack, M. (1995). Neuropsychological assessment. New York: Oxford University Press.

14. Miller, L.M., \& Lachman, M.E. (2000) Cognitive performance and the role of control beliefs in midlife. Aging, Neuropsychology and Cognition, 7, 69-85.

15. Mobility, K; Lemke, J., Grisisn G. (1991). The idea of leisure repertoire. Journal of Applied Gerontology 10(2): 208-223.

16. Pearman A. \& Storandt M. (2004). Predictors of subjective memory in older adults. Journal of Gerontology 59 (1): 4-6 
17. Perlmutter, M.(1978). What is memory aging the aging of? Developmental Psychology 14 (4): 330-345.

18. Pérez, E., Beltramino, C., Cupani, M. (2003). Inventario de autoeficacia para inteligencias múltiples: fundamentos teóricos y estudios psicométricos. Evaluar, (3), $36-60$

19. Rebok, G.W. \& Balcerak, L.J. (1989). Memory self-efficacy and performance differences in young and old adults: The effect of mnemonic training. Developmental Psychology, 25(5), 714-721.

20. Rey, M. Blasco, T \& Borràs, X. (2000). Efectos de un procedimiento de inducción de estados de ánimo sobre la autoeficacia. Anales de Psicología 16(1) 23-31.

21. Spreen, O. y Strauss E. (1998). A compendium of neuropsychological tests. New York: Oxford University Press.

22. Verhaeghen, P, Geraerts N., Marcoen. A (2000). Memory complaints, coping, and well-being in old age: a systemic approach. The Gerontologist 40, (5): 540-548.

23. West R.L. \& Yessuda M. (2004). Aging and Memory control beliefs: Performance in relation to goal setting and memory self evaluation. Journal of Gerontology: Psychological Sciences, 59 B (2): 56-65. 